\title{
Stimulation on the Positive Phase of Hippocampal Theta Rhythm Induces Long-Term Potentiation That Can Be Depotentiated by Stimulation on the Negative Phase in Area CA1 In Vivo
}

\author{
Christian Hölscher ${ }^{1}$, Roger Anwyl, ${ }^{2}$ and Michael J. Rowan ${ }^{1}$ \\ Departments of ${ }^{1}$ Pharmacology and Therapeutics and ${ }^{2}$ Physiology, Trinity College Dublin, Dublin 2, Ireland
}

Long-term potentiation (LTP) of synaptic transmission induced by high-frequency stimulation (HFS) is considered to be a model for learning processes; however, standard HFS protocols consisting of long trains of HFS are very different from the patterns of spike firing in freely behaving animals. We have investigated the ability of brief bursts of HFS triggered at different phases of background theta rhythm to mimic more natural activity patterns. We show that a single burst of five pulses at $200 \mathrm{~Hz}$ given on the positive phase of tail pinch-triggered theta rhythm reliably induced LTP in the stratum radiatum of the hippocampus of urethane-anesthetized rats. Three of these bursts saturated LTP, and 10 bursts occluded the induction of LTP by long trains of HFS. Burst stimulation on the negative phase or at zero phase of theta did not induce LTP or long-term depression. In addition, stimulation with 10 bursts on the negative phase of theta reversed previously established LTP. The results show that the phase of sensory-evoked theta rhythm powerfully regulates the ability of brief HFS bursts to elicit either LTP or depotentiation of synaptic transmission. Furthermore, because complex spike activity of approximately five pulses on the positive phase of theta rhythm can be observed in freely moving rats, LTP induced by the present theta-triggered stimulation protocol might model putative synaptic plastic changes during learning more closely than standard HFS-induced LTP.

Key words: hippocampus; CA1; long-term potentiation (LTP); depotentiation; long-term depression (LTD); theta rhythm; rhythmic slow activity; learning and memory
Long-term potentiation (LTP) of neuronal synaptic responses has been discussed for 30 years as a possible model for synaptic changes that occur during learning (Lømo, 1966; Bliss and Lømo, 1973; McNaughton et al., 1986; Morris, 1989; Bliss and Collingridge, 1993). The standard protocol used by most researchers to induce LTP is high-frequency stimulation (HFS) in which several hundred pulses at frequencies of $100-400 \mathrm{~Hz}$ are given to induce LTP. Such stimulation patterns are quite different from naturally occurring firing patterns of neurons. Endogenous neuronal firing patterns such as complex spike activity are observed in area CA1 as high-frequency bursts of approximately two to seven spikes (Buzsáki, 1986; Muller and Kubie, 1989; Otto et al., 1991). One strategy to attempt to induce LTP in a more physiological way is to give short bursts of stimuli with an interburst interval of $\sim 200 \mathrm{msec}$, called theta-patterned stimulation (Rose and Dunwiddie, 1986; Stäubli and Lynch, 1987; Diamond et al., 1988; Hölscher et al., 1997c). Such high-frequency bursts of approximately five pulses resemble complex spike activity that occurs predominantly on the positive or negative phase of theta waves (Buzsáki, 1986; Otto et al., 1991; Stewart et al., 1992; Jeffery et al., 1996). The interburst interval of $\sim 200 \mathrm{msec}$ was used to mimic the period of theta that has a dominant frequency of $\sim 3-7 \mathrm{~Hz}$ (Green and Greenough, 1986).

Single pulse stimulation with a theta-like interval ( $\sim 200 \mathrm{msec})$ when applied after LTP induction can induce depotentiation (DP), a reduction of previously potentiated EPSPs back to base-

\footnotetext{
Received March 12, 1997; revised May 22, 1997; accepted June 6, 1997.

This work was supported by the Health Research Board of Ireland, the Wellcome Trust, and the European Union.

Correspondence should be addressed to Dr. Christian Hölscher, Department of Pharmacology and Therapeutics, Trinity College Dublin, Dublin 2, Ireland.

Copyright (C) 1997 Society for Neuroscience $0270-6474 / 97 / 176470-08 \$ 05.00 / 0$
}

line levels (e.g., Stäubli and Lynch, 1990; Doyle et al., 1997). Such stimulation is at least as effective (Doyle et al., 1996; Stäubli and Chun, 1996a,b) as the standard low-frequency stimulation (LFS) protocol of $1 \mathrm{~Hz}$ for 15 min (Dudek and Bear, 1993).

Theta-patterned stimulation only imitates theta wave frequency and does not take into account the possible importance of different phases of theta activity. To test the possibility that theta phase may regulate the induction of plasticity, the effects of stimulation at different phases of artificially induced theta activity have been investigated both in vivo (Pavlides et al., 1988) and in vitro (Huerta and Lisman, 1995). Stimulation with 10 bursts of five pulses at 400 $\mathrm{Hz}$ on the positive phase of electrically evoked theta rhythm in anesthetized rats has been reported to induce LTP in the dentate gyrus, although of only relatively small magnitude (Pavlides et al., 1988). The same stimulation on the negative phase of theta had no effect on baseline but reversed LTP, although not reliably. More robust theta-triggered LTP was observed in the CA1 area of the hippocampal slice (Huerta and Lisman, 1995). LTP was induced by four pulses at $100 \mathrm{~Hz}$ given on the positive phase of carbacholinduced theta rhythm. The same burst on the negative phase did not induce long-term depression (LTD) but induced DP of previously potentiated EPSPs.

The present study investigated the possibility that the ability to induce plasticity in the CA1 area in the hippocampus may be strongly regulated by theta activity that has been triggered by sensory inputs. We used tail-pinch to trigger theta activity in urethane-anesthetized rats (e.g., Green and Greenough, 1986; Dickson et al., 1994). The application of brief bursts of stimuli (five pulses at $200 \mathrm{~Hz}$ ) on different phases of tail-pinch-evoked theta rhythm should mimic naturally occurring neuronal activity much more closely than either standard HFS or LFS. 


\section{MATERIALS AND METHODS}

Male Wistar rats weighing 200-250 gm were anesthetized with urethane (ethyl carbamate, $1.5 \mathrm{gm} / \mathrm{kg}$, i.p.) for the duration of all experiments.

Single pathway recordings of field EPSPs were made from the CA1 stratum radiatum of the right hippocampal hemisphere in response to stimulation of the Schaffer collateral/commissural pathway using techniques similar to those described previously (Doyle et al., 1996, 1997). Electrode implantation sites were identified using stereotaxic coordinates relative to bregma, with the recording site located $3 \mathrm{~mm}$ posterior and 2 $\mathrm{mm}$ right of the midline, and the stimulating electrode $4 \mathrm{~mm}$ posterior to bregma and $3 \mathrm{~mm}$ right of the midline. Bipolar stimulating and monopolar recording electrodes consisted of two pieces of twisted tungsten wire (50/75 $\mu \mathrm{m}$ inner/outer diameter; only one wire of the recording electrode was connected) insulated along its length with a Teflon coat except at the tips and were attached to a miniature connecting socket. The electrodes were slowly lowered through the cortex and the upper layers of the hippocampus into the CA1 region to a depth of $\sim 2.2 \mathrm{~mm}$ below the cortex surface and were optimally located in the stratum radiatum using electrophysiological criteria (Leung, 1980). The electrodes were then fixed in place with cyanoacrylate glue and acrylic dental cement for the stimulation and recording of evoked field EPSPs. Stainless steel screws fixed to the skull served as ground (anterior $7 \mathrm{~mm}$, lateral $5 \mathrm{~mm}$ ) and reference (posterior $8 \mathrm{~mm}$, lateral $1 \mathrm{~mm}$ ) electrodes.

All recording and stimulating was performed using an on-line computerized oscilloscope and data analysis interface system. In all experiments test EPSPs were evoked at a frequency of $0.033 \mathrm{~Hz}$, and an input-output (I/O) curve (stimulus intensity vs EPSP amplitude) was plotted for each experiment at this test frequency. For the test EPSPs, the stimulation intensity was adjusted to give an EPSP amplitude of $70 \%$ of maximum. Unless stated otherwise, the intensity was increased to give an EPSP of $90 \%$ maximum amplitude during the stimulation used to induce LTP/DP, because preliminary studies indicated that the results were more reliable when the conditioning stimulation was $>70 \%$.

To allow us to phase lock stimulation to theta wave activity, the background electroencephalogram (EEG), recorded with the same electrode as that used to monitor the EPSPs, was filtered to exclude frequencies below 1.5 and above $7 \mathrm{~Hz}$ using an analog filter unit. The filtered EEG was then sent to a phase and amplitude adjustable trigger unit (built at Trinity College by Mr. B. Ryan) that consisted of a switchable inverting/noninverting amplifier and a variable comparator serving to activate the trigger pulse. This triggered a programmable stimulating unit that in turn activated a constant current stimulus isolation unit within 0.2 msec of detecting the selected phase and level of theta wave. The data acquisition system was triggered simultaneously to record all events. Sampling speed was at $20 \mathrm{kHz}$ for baseline recording of EPSPs and $1 \mathrm{kHz}$ during recording of EEGs. The EEG was also simultaneously monitored during experiments using a digital storage oscilloscope. Theta activity was triggered by pinching the base of the rat's tail in a manner similar to that described previously (Green and Greenough, 1986; Dickson et al., 1994). Stable theta rhythm was observed in most animals after a delay of $\sim 5 \mathrm{sec}$. A burst of five pulses at $200 \mathrm{~Hz}$ was triggered on the positive or negative phase, or at zero amplitude of theta rhythm (see Fig. $1 D)$. Unless stated otherwise, the interburst interval was $\sim 1.5 \mathrm{sec}$. We also performed an experiment in which either 3 or 10 bursts were triggered on consecutive theta waves with stimulation voltage decreased to give an EPSP of $30 \%$ maximum amplitude (see Figs. $1 E, 6 c$ ). Our standard HFS protocol for inducing LTP consisted of 10 trains of 20 stimuli, interstimulus interval $5 \mathrm{msec}(200 \mathrm{~Hz})$, intertrain interval $2 \mathrm{sec}$. Three sets of this standard HFS (interset interval of $5 \mathrm{~min}$ ) induces maximal LTP under our recording conditions (Doyle et al., 1996; Hölscher et al., 1997a). LTP was measured as percentage of baseline EPSP slope recorded over the $20 \mathrm{~min}$ period before the conditioning stimulation.

Unless stated otherwise, all data are expressed as mean \pm SEM percentage baseline EPSP slope. Results were analyzed by Student's $t$ test or Welch $t$ test, which does not assume equal SDs between data sets. Theta power results were analyzed with two-way repeated measures ANOVA using a computer program (SYSTAT) after checking for normality of distribution of data.

\section{RESULTS}

\section{Theta rhythm}

The EEG was monitored in animals before and after tail pinch to establish the parameters for the onset and dynamics of theta rhythm. Figure $1 A$ shows sample EEG traces before and after tail pinch. Tail pinch caused the disappearance of large amplitude slow-wave activity and triggered theta rhythm. Figure $1 B$ shows examples of fast Fourier transformations of the EEG before and after tail pinch. A shift of the maximal power in the spectrum from $1-2$ to $4-5 \mathrm{~Hz}$ is apparent. This shift in EEG frequency after tail pinch in urethane-anesthetized rats is similar to results published by Green and Greenough (1986). Figure $1 C$ shows the power distribution of EEG measurements. Values are expressed as percentage of total power distributed over a 1-5 Hz spectrum. Twoway repeated measure ANOVA showed a difference between groups $\left(F_{(1,5)}=19.2 ; p<0.001\right)$ and power distribution $\left(F_{(9,45)}=\right.$ 13.6; $p<0.001), n=10$. Post hoc repeated measures $t$ tests showed differences between groups at $1,2,4$, and $5 \mathrm{~Hz}(1 \mathrm{~Hz}: t=3.95, p<$ 0.01; $2 \mathrm{~Hz}: t=4.0, p<0.01$; Hz: $t=4.5, p<0.01$; $5 \mathrm{~Hz}: t=3.8$, $p<0.01)$. Figure $1 d$ shows examples of burst stimulation using the conditioning pulse intensity ( $90 \%$ of maximum response) on the positive, zero, or negative phase of theta rhythm. There was a transient $(<1.5 \mathrm{sec})$ interference with the ability to record theta rhythm, especially when the stimulation was applied on the positive or zero phase of theta. Because stimulation on the negative phase had less effect, it was possible to apply repeated bursts on consecutive waves using a weak stimulation intensity (30\% of maximum response) (Fig. $1 E$ ). In this case theta rhythm was only slightly affected, with a small increase in peak-to-peak amplitude after some bursts. The latter protocol was used only in the experiment described in Figure $6 c$.

\section{Brief burst stimulation on the positive phase of theta rhythm induced LTP}

Conditioning stimulation with a single burst of five pulses on the positive phase of theta rhythm induced LTP in six of six animals tested (Fig. 2). The increase in the slope of the test EPSP was rapid in onset ( $<3 \mathrm{~min}$ ) and was stable over the recording period, measuring $119 \pm 6 \%(t=3.5 ; p<0.01)$ at $60 \mathrm{~min}$ after the conditioning stimulation. Application of three bursts of five stimuli per burst on the positive phase of theta induced LTP of $152 \pm$ $9 \%$, measured 60 min after stimulation $(t=23.5$; six of six animals; $p<0.001$ ) (Fig. $3 a$ ). As can be seen from a typical I/O curve shown in Figure $3 b$, the increase of the EPSP was present over a wide range of stimulation intensities and not just the standard test pulse intensity (70\% maximum EPSP). Indeed, when the magnitude of LTP was measured at a stimulation intensity that evoked a test EPSP that was one-third of maximum, the increase measured $\sim 200 \%$.

The LTP induced by three bursts appeared to be maximal, because 10 bursts of five stimuli elicited a similar magnitude LTP $(156 \pm 11 \%$ when measured 40 min after stimulation; $t=31.2$; six of seven animals; $p<0.001$ ) (Fig. $4 a$ ). Moreover, a standard HFS of 10 trains of 20 stimuli, interstimulus interval $5 \mathrm{msec}(200 \mathrm{~Hz})$, intertrain interval $2 \mathrm{sec}$ (in the presence of theta rhythm) did not increase LTP any further (Fig. 4a).

The effect of inducing LTP by standard HFS was investigated to compare the relative effectiveness of this stimulation with brief burst stimulation on the positive phase of theta. When a saturating amount of standard HFS was applied in the absence of theta activity, it induced an LTP to $168 \pm 16 \%$ measured 40 min later $(t=21.5$; six of six animals; $p<0.001)$ (Fig. $4 b)$. This was not significantly different from the maximum magnitude of LTP induced by brief burst stimulation on the positive phase of theta activity. 
A
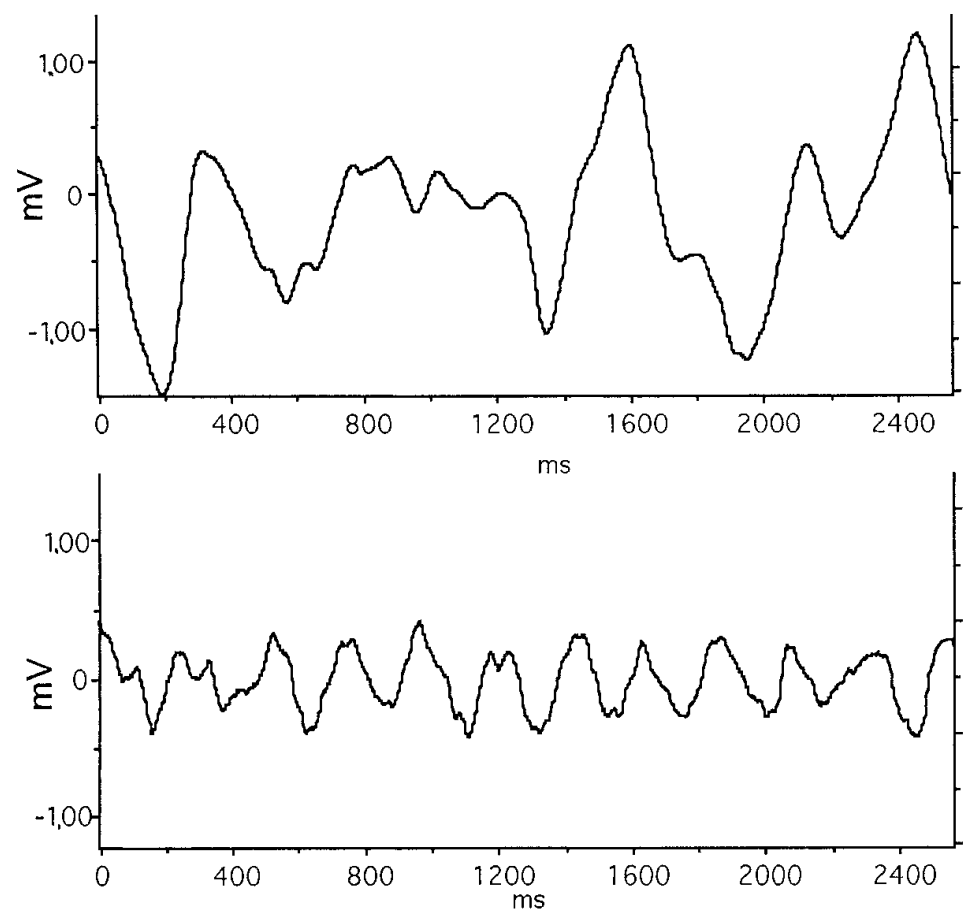

B
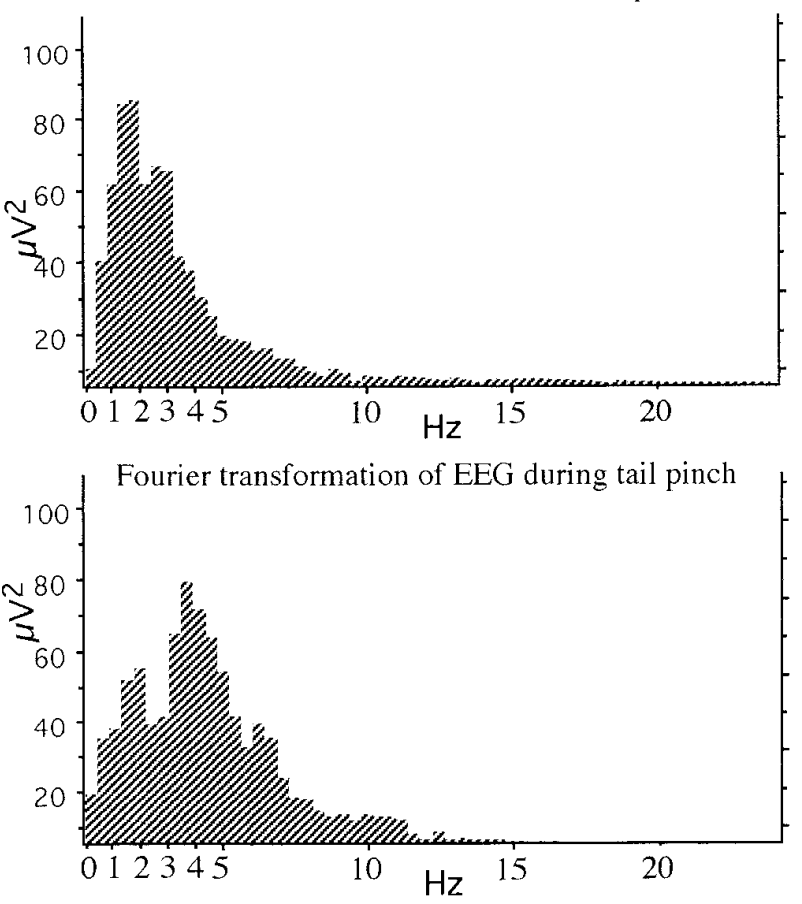

C

Relative power $(1-5 \mathrm{~Hz})$ rat EEG

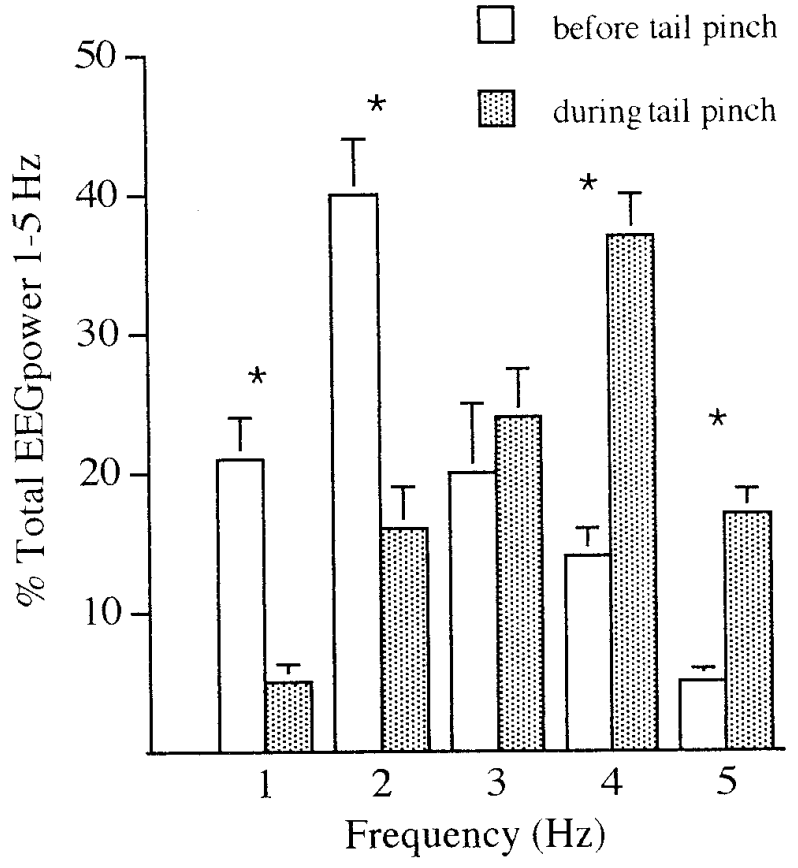

Figure 1. EEG in the dorsal hippocampus of urethane-anesthetized rats. $A$, Samples of EEGs before $(A)$ and during $(B)$ tail pinch. Although lower frequencies with large amplitudes were suppressed after the tail pinch, higher frequencies of $\sim 4 \mathrm{~Hz}$ (theta) with a very regular cycle appeared. B, Examples of Fourier transformation of EEGs before (top) and during (bottom) tail pinch. A shift of the power distribution from 1-2 to $3-4 \mathrm{~Hz}$ was visible. $C$, The power distribution of EEG measurements taken before and during tail pinch. Values are expressed as percentage of total power distributed over $1-5 \mathrm{~Hz}$ spectrum. ${ }^{*} p<0.01 . D$, Sample traces of stimulation on the positive $(a)$, zero (neutral) $(b)$, or negative $(c)$ phase of theta rhythm. The left scale shows theta wave amplitude, and the right scale shows stimulus intensity as measured on a different channel. Theta rhythm was induced by tail pinch, and a burst of five pulses at $200 \mathrm{~Hz}$ was triggered at a preset amplitude level of theta rhythm. Note that subsequent theta rhythm was disturbed by the stimulation. The large amplitude wave in the EEG immediately after the stimulation is composed of the summated electrically evoked EPSPs. $E$, Stimulation on the negative phase of theta rhythm on 10 consecutive theta waves. In this protocol the stimulation intensity was reduced (30\% of maximum EPSP response) to avoid disturbance of theta rhythm as shown in $d$. The left scale shows theta wave amplitude, and the right scale shows stimulus intensity as measured on a different channel. Figure continues.
Brief burst stimulation on the negative or zero phase of theta rhythm or in its absence did not induce LTP or LTD

Stimulation with 3 or 10 bursts at zero phase of theta rhythm did not induce a change in the slope of EPSP (six of six animals) (Fig. $5 a$ ). Furthermore, stimulation with 3 or 10 bursts on the negative phase of theta rhythm did not induce LTP or LTD (six of six animals) (Fig. 5b). Stimulation with three bursts of five pulses per burst in the absence of tail pinch-triggered theta activity also had no long-lasting effect on synaptic transmission. Thus the slope of the test EPSP measured $104 \pm 5 \% 60$ min after stimulation (six of six animals; graph not shown).

\section{Brief burst stimulation on the negative phase of theta rhythm induced DP}

In these experiments, large amplitude LTP was first induced by stimulating with three bursts on the positive phase of theta waves. 

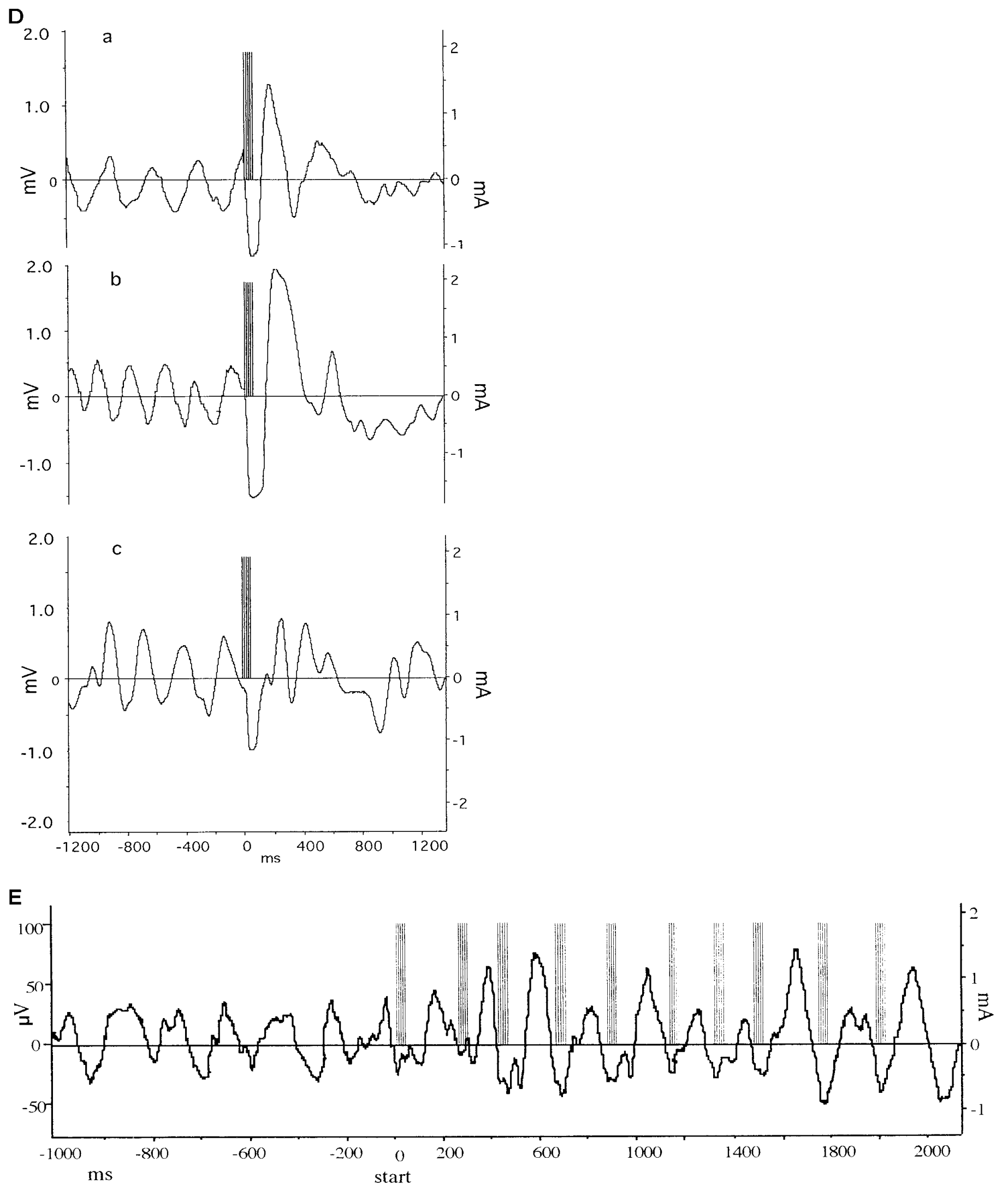

Figure 1 continued. 


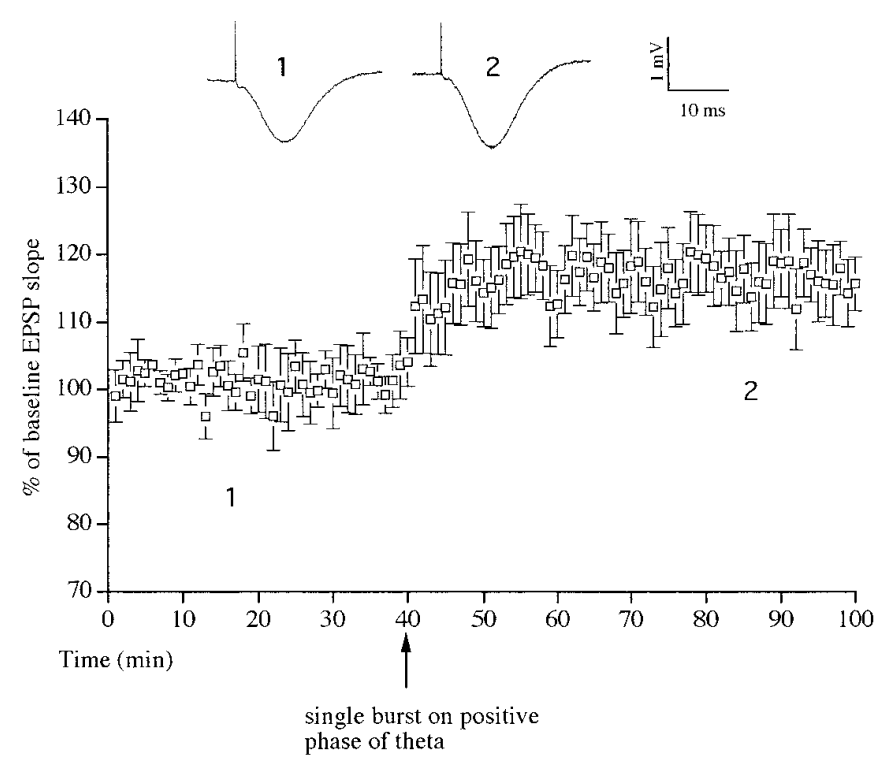

Figure 2. Stimulation with a single burst of five pulses at $200 \mathrm{~Hz}$ on the positive phase of theta rhythm induced LTP. The LTP was stable during the 60 min recording period $(p<0.01 ; n=6)$. Single traces of average field EPSPs are shown as numbered insets. The numbers refer to times of sampling shown in the graph.

Thirty minutes later burst stimulation was applied on the negative or zero phase of theta to attempt to induce DP.

In one set of experiments, strong stimuli (90\% of maximum EPSP response) were applied on the negative phase of theta with an interburst interval of $1.5 \mathrm{sec}$. Stimulation with three bursts on the negative phase of theta $30 \mathrm{~min}$ after inducing LTP (to $146 \pm$ $8 \% ; t=20.5$; six of six animals; $p<0.001$ ) induced a short-term depression of only $10 \%$, but no DP was induced (six of six animals) (Fig. $6 a$ ); however, stronger stimulation on the negative phase of theta rhythm did induce depotentiation (DP). As shown in Figure $6 b$, LTP of $142 \pm 7 \%(t=17.8$; six of six animals; $p<$ $0.001)$ was depotentiated to $110 \pm 8 \%$ when stimulation with 10 bursts was applied on the negative phase of theta $(t=5.8$; six of six animals; $p<0.001$; measured 30 min after negative phase stimulation). LTP was restored by subsequent stimulation with three bursts on the positive phase of theta (to $140 \pm 7 \%$ when measured 30 min later; $t=16.8$; six of six animals; $p<0.001$ ) (Fig. 6b). In contrast, stimulation with 10 bursts on the zero phase of theta $30 \mathrm{~min}$ after the induction of LTP did not induce DP (from $154 \pm 7 \%$ LTP to $151 \pm 11 \% 20 \mathrm{~min}$ after zero phase burst stimulation; $n=4)$.

In a separate set of experiments, conditioning stimulation was applied at a weaker intensity (30\% maximum response, an intensity that did not disrupt theta) on the negative phase of consecutive theta waves. Three mild bursts on consecutive theta waves did not induce DP (from $159 \pm 3 \%$ to $153 \pm 5 \%$ of baseline 20 min after stimulation; $n=6$; data not shown). Stimulation on the negative phase of 10 consecutive theta waves (Fig. $1 E$ ), however, induced DP of previously potentiated EPSPs from $161 \pm 9 \%$ of baseline values down to $120 \pm 4 \%$ when measured 30 min later $(t=14.9$; six of six animals; $p<0.001)$. LTP was restored by subsequent stimulation with three bursts on the positive phase of theta waves (to $143 \pm 7 \%$ when measured 30 min later; $t=15.2$; six of six animals; $p<0.001$ ) (Fig. $6 c$ ).

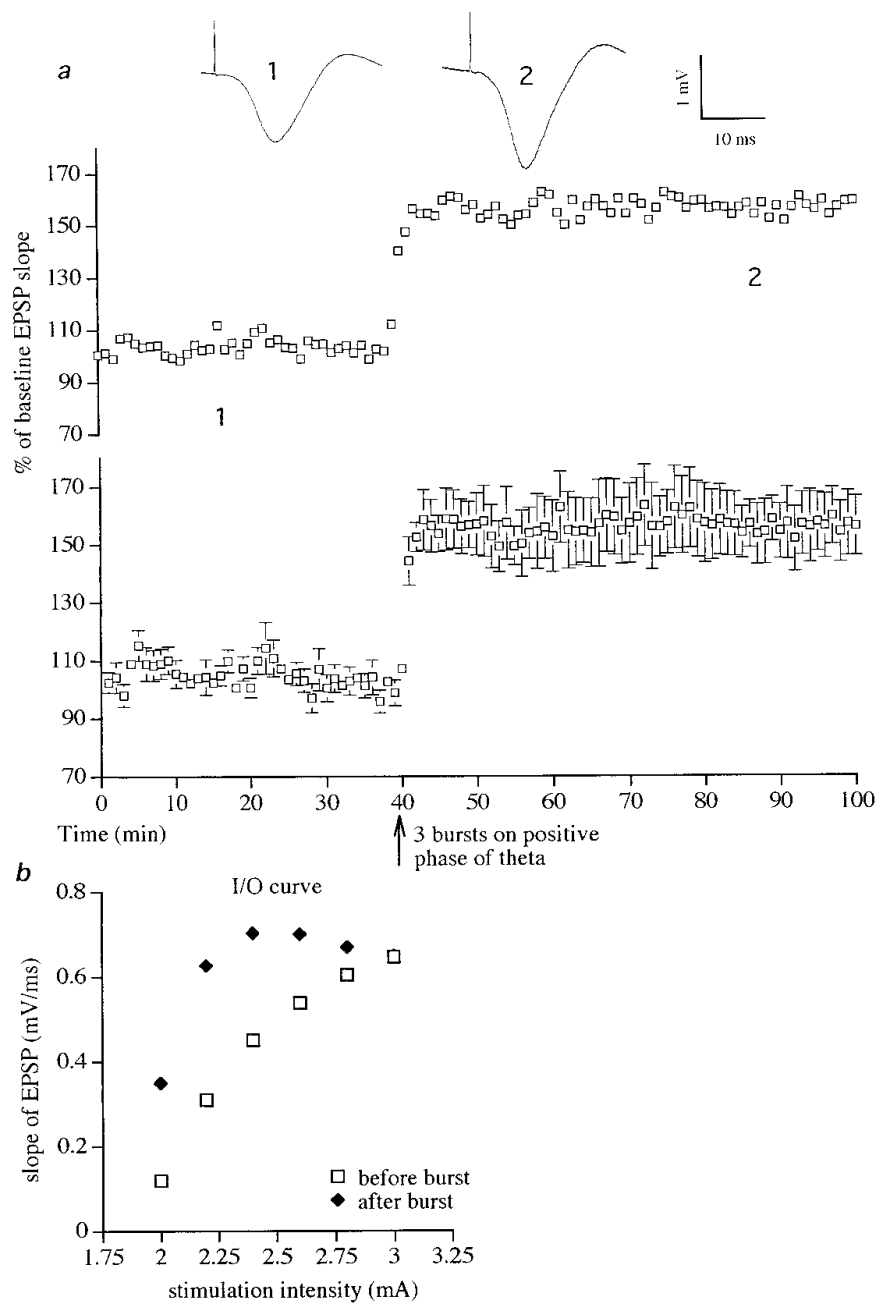

Figure 3. Stimulation with three bursts (five pulses per burst) on the positive phase of theta rhythm induced large LTP. $a$, The top graph shows data from a single animal, whereas the bottom graph is the average of six experiments $(p<0.001)$. Single traces of average field EPSPs are shown as numbered insets. The numbers refer to times of sampling shown in the graph. $b$, Example of an I/O curve (stimulus intensity vs amplitude of field EPSP) before and after stimulation on the positive phase of theta rhythm. The largest potentiation was observed at a stimulation intensity that evoked an EPSP one-third to one-fifth of the maximum slope.

\section{DISCUSSION}

The present study shows for the first time that LTP can be reliably induced in the CA1 area of the intact hippocampus with as few as five pulses when applied as a single burst at 200 $\mathrm{Hz}$ on the positive phase of sensory-evoked theta. In contrast, brief burst stimulation had no effect when given on the negative or zero phase or in the absence of theta. LTP was saturated with as few as three bursts of five pulses on the positive phase of theta. Theta-triggered LTP occluded standard HFSinduced LTP and had a maximum amplitude that was equivalent to the maximum induced by standard HFS.

Our data are consistent with and greatly extend the findings of a previous in vivo experiment performed in the dentate gyrus of urethane-anesthetized rats (Pavlides et al., 1988). In that study a small (9\%) LTP of the EPSP was induced when 10 bursts (five pulses at $400 \mathrm{~Hz}$ ) were applied on the positive phase of electrically induced theta rhythm. This suggests that 

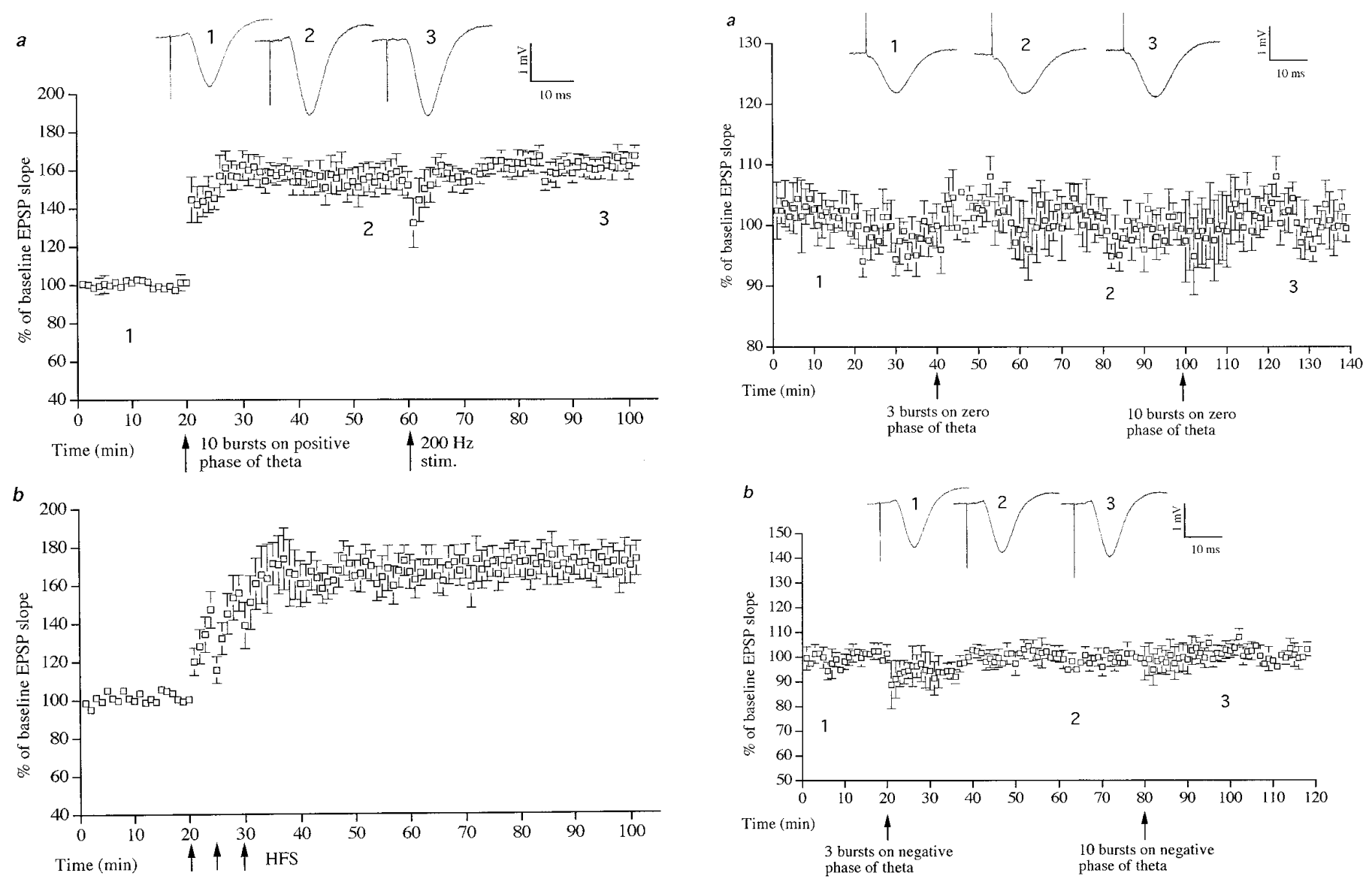

Figure 4. LTP induced with theta-triggered stimulation occluded HFSinduced LTP. $a$, Stimulation with 10 bursts on the positive phase of theta rhythm induced LTP $(p<0.001 ; n=6)$. Additional stimulation with 200 pulses at $200 \mathrm{~Hz}$ did not increase the slope of EPSPs any further. $b$, For comparison, maximal HFS-induced LTP is shown $(n=6)$. There is no difference between LTP induced by HFS or by bursts on the positive phase of theta rhythm. Single traces of field EPSPs from the burstinduced LTP group are shown. The numbers refer to times of sampling shown in the graph.

there is an inherent difference in the inducibility of LTP in the CA1 area and the dentate gyrus in response to theta-triggered stimulation. A previous in vitro study in area CA1 found that LTP was induced by a single burst of four pulses at $100 \mathrm{~Hz}$ given on the positive phase of carbachol-induced theta rhythm (Huerta and Lisman, 1995; Lisman, 1997). There is doubt, however, about whether carbachol-induced theta activity accurately mimics naturally occurring theta, such as that used in the present experiments (Stewart and Fox, 1990).

The high sensitivity of LTP to positive phase stimulation is reminiscent of the increased responsiveness of LTP induction to theta-patterned stimulation. In particular, Diamond et al. (1988) showed that a burst of four pulses, which were preceded by a single pulse $200 \mathrm{msec}$ previously, induced stable LTP in the intact hippocampus. This priming is thought to be attributable to the presence of minimal inhibition at this time interval (Diamond et al., 1988). Because theta activity is known to be associated with oscillations in the level of inhibitory neuron activity (Vanderwolf and Leung, 1983; Buzsáki, 1986), somewhat similar mechanisms may be responsible for the ability to induce LTP with a single burst on the positive phase of theta in the present study. We have shown previously, however, that a theta-patterned stimulation protocol

Figure 5. Stimulation on the zero or negative phase of theta did not induce LTP or LTD. $a$, Stimulation with 3 or 10 bursts at zero phase of theta rhythm did not alter EPSPs $(n=6)$. $b$, Stimulation with 3 or 10 bursts on the negative phase of theta rhythm did not change EPSPs $(n=$ $6)$. Single traces of field EPSPs are shown. The numbers refer to times of sampling shown in the graphs.

(five pulses per burst, $200 \mathrm{msec}$ interburst interval, in the absence of theta) under the same experimental conditions as described here required a minimum of four consecutive bursts to induce stable LTP (Hölscher et al., 1997c). It would appear therefore that stimulation on the positive phase of theta rhythm is a more effective method of inducing LTP than at least some theta-patterned stimulation protocols.

In view of our finding that theta-triggered LTP occluded HFSinduced LTP, it will be of interest to compare their pharmacological profiles to find out whether both forms of LTP are based on activation of similar receptors and biochemical processes. In a previous in vitro study, Huerta and Lisman (1995) reported that LTP induced by stimulation on the positive phase of theta was blocked by NMDA glutamate receptor and muscarinic acetylcholine receptor antagonists at concentrations that inhibited theta activity. It is known that theta activity in vivo can be blocked by such agents (Bland, 1986; Leung and Desborough, 1988; Dickson et al., 1994).

Remarkably, stimulation on the negative phase of theta reversed previously established LTP. Thus, the application of 10 bursts of five pulses at $200 \mathrm{~Hz}$ on the negative phase of tail-pinch-evoked theta activity, 30 min after LTP induction, resulted in a large DP. Stimulation on the zero phase of theta was without effect. These results are much more convincing than those reported previously 

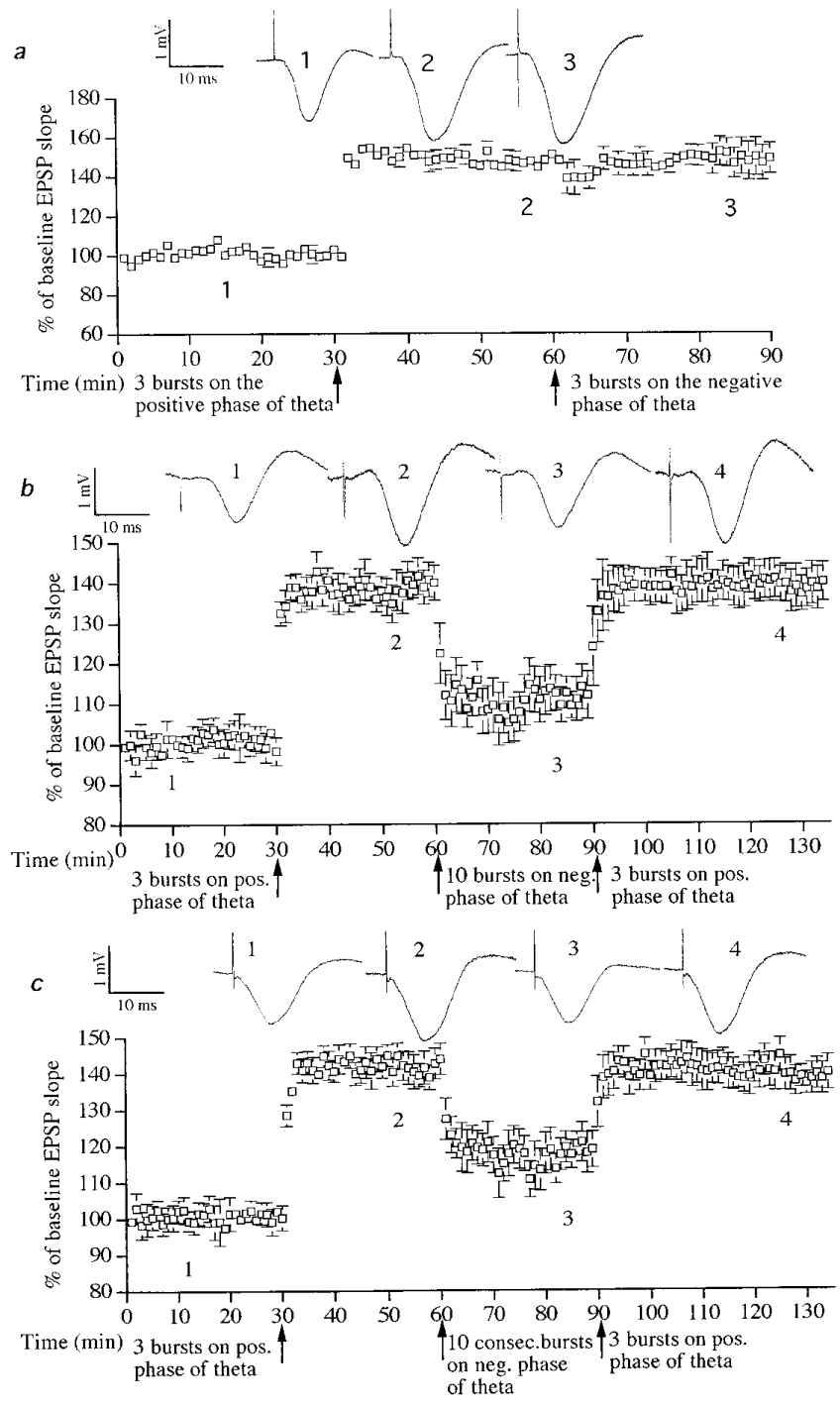

Figure 6. Stimulation with 10 bursts on the negative phase of theta induced DP. $a$, Stimulation with three bursts on the negative phase of theta rhythm did not reverse previously established LTP $(n=6)$. $b$, Stimulation with three bursts on the positive phase of theta waves induced LTP $(p<$ $0.001 ; n=6$ ). Subsequent stimulation 30 min later with 10 bursts (interburst interval of $1.5 \mathrm{sec})$ on the negative phase of theta waves induced DP ( $p<$ $0.001)$. Stimulation with three bursts on the peak of theta waves restored LTP $(p<0.001) . c$, Stimulation with 10 bursts on 10 consecutive troughs of theta waves using weaker stimulus intensity (see Fig. $1 E$ ) also induced DP $(p<0.001)$. Sample EPSPs before and after stimulation are shown. Numbers refer to sampling times indicated in the graph.

for electrically triggered theta in the dentate gyrus in vivo (Pavlides et al., 1988). In that study, stimulation with 10 bursts of five pulses at $400 \mathrm{~Hz}$ induced DP in only three of seven experiments. In contrast, in area CA1 in vitro a single burst of four pulses on the negative phase of carbachol-induced theta induced DP (Huerta and Lisman, 1995). Why much stronger stimulation was needed in the in vivo experiments described here is not clear. It is possible that carbachol can directly facilitate the induction of DP in addition to its modulatory action via theta activity (Barkai and Hasselmo, 1995; Kirkwood et al., 1996). Alternatively, the use of urethane may be responsible for the difference. It is interesting to note that burst stimulation interfered with theta rhythm to the least extent when relatively weak stimulation ( $30 \%$ of maximum) was used on the negative phase of theta (Fig. $1 E$ ). In this case it was possible to stimulate on the trough of consecutive theta waves without disrupting theta rhythm. Such stimulation had an interburst interval of $\sim 200 \mathrm{msec}$ (as opposed to an interburst interval of $1.5 \mathrm{sec}$ with strong stimuli) but resulted in an equivalent magnitude of DP. Obtaining DP with 10 bursts of five pulses is significantly less than the number of stimuli required to induce DP with LFS in most previous studies; usually $300-900$ pulses at $1-10 \mathrm{~Hz}$ are necessary (Stäubli and Lynch, 1990; O'Dell and Kandel, 1994; Doyle et al., 1996; Stäubli and Chun, 1996a; Hölscher et al., 1997a; but see Stäubli and Chun, 1996b). It appears therefore that theta rhythm facilitated the induction of DP. Although the mechanisms of DP induction are poorly understood, previous reports indicate that excitatory stimulation at a time when there was strong inhibition of pyramidal cell firing (using a paired-pulse protocol) enabled the induction of LTD in vivo (Thiels et al., 1995; Doyère et al., 1996). Earlier studies suggest that the neuronal system is under greater inhibition on the negative phase of theta (Buzsáki, 1986). Thus stimulation with excitatory bursts during a period of relative inhibition may account for the observed facilitation of DP induction. Overall, the finding that the same brief burst stimulation on different phases of theta could induce LTP or DP indicates that the phase of sensory-evoked theta rhythm regulates not only the threshold for hippocampal plasticity but also its direction.

LTD was not observed at all in the present study. This is consistent with a large number of previous in vivo studies in which DP but not LTD was readily inducible (Pavlides et al., 1988; Stäubli and Lynch, 1990; Doyle et al., 1996, 1997; Hölscher et al., 1997a; but see Heynen et al., 1996). Furthermore, it was reported recently that although LTD could not be induced with LFS in the CA1 area of nonstressed adult animals, in stressed animals LTD was easily induced (Kim et al., 1996; Xu et al., 1997).

It is impressive that LTP could be reliably induced in the CA1 area of the intact hippocampus with as few as five pulses when given on the positive phase of sensory-evoked theta. The finding that LTP and DP induction is dependent on theta rhythm suggests that these forms of synaptic plasticity play a role in cognitive processes. Theta rhythm occurs naturally during motor activity or novelty perception and is believed to be important for memory formation, because the blocking of theta rhythm impairs the ability of rats to learn a spatial task (Winson, 1978). Furthermore, a stimulation pattern of one burst of five pulses at $200 \mathrm{~Hz}$ resembles natural firing patterns such as complex spike activity more closely than long trains of HFS or LFS. It has been suggested that complex spike activity represents the activity of pyramidal neurons that process or convey sensory input (Vinogradova, 1975; Otto et al., 1991; O'Keefe and Burgess, 1996). Because theta rhythm is induced by novel stimuli and not during situations in which few potentially important sensory stimuli are present, theta rhythm could be part of a filter system that amplifies stimuli of relative importance to the animal and facilitates the induction of LTP/DP-like processes. It can be imagined that complex spike activity represents or encodes sensory information. Selective synaptic changes of activated synapses or neurons could form a memory trace of neuronal activity patterns for future reference (Burgess et al., 1996).

Recently published evidence suggests that HFS-induced LTP might not be a valid model for learning mechanisms, as had been postulated previously (Bannerman et al., 1995; Huang et al., 1995; Saucier and Cain, 1995; Nosten-Bertrand et al., 1996; Hölscher et al., 1997a,b). Whether LTP induced by brief burst stimulation on the positive phase of theta wave is correlated with learning and only with learning and not with other factors such as novelty 
detection or motor activity needs to be tested in freely moving rats. The encouraging results presented here, however, show that LTP induction is facilitated by theta activity, and this might be a facilitation mechanism that is active during learning. LTP obtained this way could model neuronal changes that may underlie memory formation better than HFS-induced LTP.

\section{REFERENCES}

Bannerman DM, Good MA, Butcher SP, Ramsay M, Morris RGM (1995) Distinct components of spatial learning revealed by prior training and NMDA receptor blockade. Nature 378:182-186.

Barkai E, Hasselmo ME (1995) Cholinergic enhancement of LTP and LTD in rat piriform cortex: testing predictions of a model. Soc Neurosci Abstr 22:1510.

Bland BH (1986) The physiology and pharmacology of hippocampal formation theta rhythms. Prog Neurobiol 26:1-54.

Bliss T, Lømo T (1973) Long-lasting potentiation of synaptic transmission in the dentate area of the anaesthetised rabbit following stimulation of the perforant path. J Physiol (Lond) 232:331-356.

Bliss TVP, Collingridge GL (1993) A synaptic model of memory: longterm potentiation in the hippocampus. Nature 361:31-39.

Burgess N, Recce M, O'Keefe J (1996) A model of hippocampal function. Neuron Netw 7:1065-1081.

Buzsáki G (1986) Generation of hippocampal EEG Patterns. In: The hippocampus (Isaacson RL, Pribram KH, eds), pp 137-167. New York: Plenum.

Diamond DM, Dunwiddie TV, Rose GM (1988) Characteristics of hippocampal primed burst potentiation in vitro and in the awake rat. J Neurosci 8:4079-4088.

Dickson CT, Trepel C, Bland BH (1994) Extrinsic modulation of theta field activity in the entorhinal cortex of the anesthetized rat. Hippocampus 4:37-51.

Doyère V, Errington ML, Laroche S, Bliss TVP (1996) Low-frequency trains of paired stimuli induce long-term depression in area CA1 but not in dentate gyrus of the intact rat. Hippocampus 6:52-57.

Doyle C, Hölscher C, Rowan MJ, Anwyl R (1996) The selective neuronal NO synthase inhibitor 7-nitro-indazole blocks both long-term potentiation and depotentiation of field EPSPs in rat hippocampal CA1 in vivo. J Neurosci 16:418-426.

Doyle C, Cullen WK, Rowan MJ, Anwyl R (1997) Low frequency stimulation induces homosynaptic depotentiation but not long-term depression of synaptic transmission in the adult anaesthetized and awake rat hippocampus in vivo. Neuroscience 77:75-85.

Dudek SM, Bear MF (1993) Bidirectional long-term modification of synaptic effectiveness of adult and immature hippocampus. J Neurosci 13:2190-2198.

Green EJ, Greenough WT (1986) Altered synaptic transmission in dentate gyrus of rats reared in complex environments: evidence from hippocampal slices maintained in vitro. J Neurophysiol 55:739-750.

Heynen AJ, Abraham WC, Bear MF (1996) Bidirectional modification of CA1 synapses in the adult hippocampus in vivo. Nature 381:163-166.

Hölscher C, McGlinchey L, Roger Anwyl R, Rowan MJ (1997a) HFSinduced long-term potentiation and depotentiation in area CA1 of the hippocampus are not good models for learning. Psychopharmacology 130:174-182.

Hölscher C, Anwyl R, Rowan M (1997b) Block of HFS-induced LTP in the dentate gyrus by 1S,3S-ACPD: further evidence against LTP as a model for learning. NeuroReport 8:451-454.

Hölscher C, Anwyl R, Rowan M (1997c) Block of theta-burst induced LTP by 1S,3S-ACPD: further evidence against LTP as a model for learning. Neuroscience, in press.

Huang YY, Kandel ER, Varshavsky L, Brandon EP, Qi M, Idzerda RL, McKnight GS, Bourtchouladze R (1995) A genetic test of the effects of mutations in PKA on mossy fiber LTP and its relation to spatial and contextual learning. Cell 83:1211-1222.

Huerta PT, Lisman JE (1995) Bidirectional synaptic plasticity induced by a single burst during cholinergic theta oscillation in CA1 in vitro. Neuron 15:1053-1063.

Jeffery KJ, Donnett JG, O'Keefe J (1996) Medial septal control of thetacorrelated unit firing in the entorhinal cortex of awake rats. NeuroReport 6:2166-2170.

Kim JJ, Foy MR, Thompson RF (1996) Behavioral stress modifies hippocampal plasticity through $N$-methyl-D-aspartate receptor activation. Proc Natl Acad Sci USA 93:4750-4753.
Kirkwood A, Kirkwood J, Perez F, Bear MF (1996) Facilitation of synaptic plasticity by ACh and NE in rat visual cortex. Soc Neurosci Abstr 23:1729.

Leung LWS (1980) Behavior-dependent evoked potentials in the hippocampal CA1 region of the rat. 1. Correlation with behavior and EEG. Brain 198:95-117.

Leung LWS, Desborough KA (1988) APV, an N-methyl-D-aspartate receptor antagonist, blocks the hippocampal theta rhythm in behaving rats. Brain Res 463:148-152.

Lisman JE (1997) Bursts as a unit of neural information: making unreliable synapses reliable. Trends Neurosci 20:38-43.

Lømo T (1966) Frequency potentiation of excitatory synaptic activity in the dentate area of the hippocampal formation. Acta Physiol Scand 68(Suppl 277):128.

McNaughton BL, Barnes CA, Baldwin RG, Rasmussen M (1986) Longterm enhancement of hippocampal synaptic transmission and the acquisition of spatial information. J Neurosci 6:563-571.

Morris RGM (1989) Synaptic plasticity and learning: selective impairment of learning in rats and blockade of long-term potentiation in vivo by the $N$-methyl-D-aspartate receptor antagonist AP5. J Neurosci 9:3040-3057.

Muller RU, Kubie JL (1989) The firing of hippocampal place cells predicts the future position of freely moving rats. J Neurosci 9:4101-4110.

Nosten-Bertrand M, Errington ML, Murphy KPSJ, Tokugawa Y, Barboni E, Kozlova E, Michalovich E, Morris RGM, Silver J, Stewart CL, Bliss TVP, Morris RJ (1996) Normal spatial learning despite regional inhibition of LTP in mice lacking Thy-1. Nature 379:826-829.

O'Dell T, Kandel E (1994) Low-frequency stimulation erases LTP through an NMDA receptor mediated activation of protein phosphatases. Learn Mem 1:83-152.

O'Keefe J, Burgess N (1996) Geometric determinants of the place fields of hippocampal-neurons. Nature 381:425-428.

Otto T, Eichenbaum H, Wiener S, Wible C (1991) Learning-related patterns of CA1 spike trains parallel stimulation parameters optimal for inducing hippocampal long-term potentiation. Hippocampus 1:181-192.

Pavlides C, Greenstein YJ, Grudman M, Winson J (1988) Long-term potentiation in the dentate gyrus is induced preferentially on the positive phase of $\theta$-rhythm. Brain Res 439:383-387.

Rose GM, Dunwiddie TV (1986) Induction of hippocampal long-term potential using physiologically patterned stimulation. Neurosci Lett 69:244-248.

Saucier D, Cain DP (1995) Spatial learning without NMDA receptordependent long-term potentiation. Nature 378:186-189.

Stäubli U, Chun D (1996a) Factors regulating the reversibility of longterm potentiation. J Neurosci 16:853-860.

Stäubli U, Chun D (1996b) Proactive and retrograde effects on LTP produced by theta pulse stimulation: mechanisms and characteristics of LTP reversal in vitro. Learn Memory 3:96-105.

Stäubli U, Lynch G (1987) Stable hippocampal long-term potentiation elicited by "theta" pattern stimulation. Brain Res 435:227-234.

Stäubli U, Lynch G (1990) Stable depression of potentiated synaptic responses in the hippocampus with $1-5 \mathrm{~Hz}$ stimulation. Brain Res 513:113-118.

Stewart M, Fox SE (1990) Do septal neurons pace the hippocampal rhythm? Trends Neurosci 13:163-168.

Stewart M, Luo Y, Fox SE (1992) Effects of atropine on hippocampal theta cells and complex-spike cells. Brain Res 591:122-128.

Thiels E, Barrionuevo G, Berger T (1995) Excitatory stimulation during postsynaptic inhibition induces long-term depression in hippocampus in vivo. J Neurophysiol 72:3009-3116.

Vanderwolf CH, Leung L-WS (1983) Hippocampal rhythmical slow activity: a brief history and the effects of entorhinal lesions and phencyclidine. In: Neurobiology of the hippocampus (Seifert W, ed), pp 275302. London: Academic.

Vinogradova OS (1975) Functional organization of the limbic system in the process of registration of information: facts and hypotheses. In: The hippocampus: 2. Neurophysiology and behavior (Isaacson RL, Pribram $\mathrm{KH}$, eds), pp 1-70. New York: Plenum.

Winson J (1972) Inter-species differences in the occurrence of theta. Behav Biol 7:479-487.

Winson J (1978) Loss of hippocampal theta rhythm results in spatial memory defects of the rat. Science 201:160-163.

Xu L, Anwyl R, Rowan MJ (1997) Behavioural stress facilitates the induction of long-term depression in the hippocampus. Nature 387:497-500. 